\title{
A Modified Q-Learning Algorithm for Robot Path Planning in a Digital Twin Assembly System
}

Xiaowei Guoa ( $\nabla$ xiaoweig@usc.edu )

University of Southern California

\section{Research Article}

Keywords: Path planning, digital twin, Q-learning, case-based reasoning

Posted Date: August 25th, 2021

DOl: https://doi.org/10.21203/rs.3.rs-825772/v1

License: (c) (i) This work is licensed under a Creative Commons Attribution 4.0 International License. Read Full License

Version of Record: A version of this preprint was published at The International Journal of Advanced Manufacturing Technology on January 10th, 2022. See the published version at https://doi.org/10.1007/s00170-021-08597-9. 
A Modified Q-learning Algorithm for Robot Path Planning in a Digital Twin Assembly System

$$
\text { Xiaowei Guo }{ }^{\mathrm{a}}
$$

Abstract: Product assembly is an important stage in complex product manufacturing. How to intelligently plan the assembly process based on dynamic product and environment information has become an pressing issue needs to be addressed. For this reason, this research has constructed a digital twin assembly system, including virtual and real interactive feedback, data fusion analysis and decision-making iterative optimization modules. In the virtual space, a modified Q-learning algorithm is proposed to solve the path planning problem in product assembly. The proposed algorithm speeds up the convergence speed by adding dynamic reward function, optimizes the initial Q table by introducing knowledge and experience through the case-based reasoning (CBR) algorithm, and prevents entry into the trapped area through the obstacle avoiding method. Finally, take the six-joint robot UR10 as an example to verify the performance of the algorithm in the three-dimensional pathfinding space. The experimental results show that the modified Q-learning algorithm's pathfinding performance is significantly better than the original Q-learning algorithm.

Keywords: Path planning; digital twin; Q-learning; case-based reasoning

\section{Introduction}

Assembly is one of the most important parts of the industrial production process ${ }^{[1]}$. With the development of nowadays technology, humans are gradually replaced by industrial robots during the assembly process.

${ }^{a}$ Daniel J. Epstein Dept. of Industrial \& Systems Engineering, University of Southern California, Los Angeles, CA 90089

E-mail: xiaoweig@usc.edu 
Meantime, the development of industrial robots has also gone through several stages. Industrial robots using traditional assembly methods can only run under a set path. People usually use Cartesian coordinates to describe the robot's posture and obtain data through some sensors, so as to obtain the relationship between the robot arms and the targets or obstacles. Then, the D-H method is used to extract the rods' parameters and joints' variables, combined with the rigid body pose description theory and the inverse transformation method to solve the positive and negative kinematics $^{[2]}$. This method requires plenty of calculation and a lot of human engagement. People have to plan the path manually for the robots, and what the robot can do is only repeatedly work on the route planned by the human, and feedback a series of displacement, velocity, and acceleration data to the human. This method will result in the robot having no way to adapt to the environment, and no way to avoid obstacles autonomously.

However, with the current economic development, customers have stricter requirements for products. This is followed by more and more complex product designs and higher demand for precision. The drawback of impotent real-time communication may lead to a negative influence on the verification of the component model as well as the evaluation of overall product assembly, which will affect the whole product quality. And the ability to autonomously avoiding obstacles becomes much more important for industrial robots. Thus, virtual assembly and machine learning were introduced. In virtual assembly, users can use various interactive devices to perform various assembly operations on product components as in reality. During the operation, the system provides real-time detection, management, and planning functions, so that users can easily analyze the feasibility of product assembly. After the process is completed, the system will take down all the information from the assembly process, and generate reports, video recordings for continued analysis. 
Since the most important parts in the virtual assembly system are collecting data and human-computer interaction, two new concepts should be introduced into this paper----digital twins and CPS (Cyber-Physical Systems). Digital twin can be considered as an interdisciplinary, multi-scale simulation process that makes full use of data such as sensors, physical models, and operating history. It mirrors the physical products into a virtual space and represents the whole life cycle of the corresponding physical products. It allows different steps of the assembly process connected with each other based on the communication and information technology, which can help reduce the deviations and as well as increase the efficiency of the entire production life cycle ${ }^{[4]}$. While, CPS, developed in the era of the Industrial Revolution 4.0, including network layer, perception layer, and control layer, integrates communications, control, and physical through the Internet ${ }^{[5]}$. The perception layer is mainly composed of sensors, controllers, and collectors. The sensor in the perception layer is the terminal device in the CPS and collects the needed information from the surrounding environment before sending the collected data to the server on a regular basis. In the later process, the terminal device will make changes in response to the data returned from the server through the physical terminal device after processing. The data transmission layer is the connecting bridge between the information world and the physical world. CPS does help the assembly systems a lot, under the situation of the increasing variety of products. It promotes the assembly systems with the ability to reconfigure, which helps them better adapt to different product variants. ${ }^{[6]}$

In order to make the robot more intelligent when dealing with complex situations in unknown environments, it's quite important to introduce the concepts of reinforcement learning and deep learning into the path planning process. Deep learning is a general term for a type of pattern analysis method that includes three types of methods: Convolutional Neural Network (CNN), the self-encoding neural network based on multi-layer neurons, and deep belief network (DBN) of the neural network weight. While reinforcement learning means that the subject learns in a "trial 
and error method", and through interaction with the environment to reward and guide behavior so that the subject can obtain the greatest reward ${ }^{[7]}$. Q-Learning, one of the commonly used algorithms in reinforcement learning, is a value-based algorithm, which is the expectation of gaining benefits by taking actions at a certain moment. The environment will feedback corresponding rewards based on the agent's actions. To sum up, the fundamental idea of this algorithm is selecting the action with the greatest benefit from the constructed Q-table, in which the data represents the state and action of the agent. With the application of Reinforcement Learning, human involvement can be reduced from the assembly system, and the robustness of the system can be increased. It also allows the assembly robot to learn complex behaviors based on interaction with the outside environment. In the path planning process, it's quite important for the agent to connect with the surrounding environment, and make the right decision on the next behavior, which means the agent can perform a high-precision assembly process instead of translating human behaviors into robot program during the path planning process. ${ }^{[8]}$

The rest of this paper is organized as follows. Section 2 reviews the related research literature. In Section 3, the digital twin framework for assembly is described. The detailed algorithm definition and improvement of the Q-learning model are analyzed in Section 4. Computational experiments of the UR10 robot are described in Section 5. Finally, conclusions are given in Section 6, together with some discussion of potential future work.

II. Related works

A. Path planning

Path planning has always been a vital part in production process. It has great influence on production efficiency and safety. Thus, how to let the industrial robots automatically plan their path has become a very important issue. Path planning can be 
divided into global path planning and local path planning. The location of the obstacles in the global path planning are known. Thus, the main mission of global path planning is to build up the model of the environment after calculation, and then optimize the path. Commonly used methods are: visible method, free space method, grid method, Dijkstra algorithm, A* algorithm. However, the accuracy of global path planning is hard to ensure under complex and changing environment. While the local path planning needs the industrial robots to collect information and data from the environment through the whole assembly process, which requires the robots to have learning ability. Artificial potential field method, fuzzy control algorithm, hierarchical algorithm, neural network algorithm and reinforcement learning method are most commonly used methods in local path planning.

Khatib et al.(1986) first introduced the path planning of artificial potential field method, a virtual force method, to the robot field, which helps create the robot movement in the surrounding environment into an abstract artificial gravitational field. This method can result in a generally smooth and safe path, while may also lead the robot to the problem of local optimum ${ }^{[9]}$. Tsong-Li Lee et al.(2003) introduced a fuzzy algorithm, which can determine the priority of several possible paths, and repeatedly drive the robot to the final configuration in the highest priority forward direction ${ }^{[10]}$. Zuo, L et al. proposed a hierarchical path planning approach, a new layered path planning method, which contain two different level of structure. A grid-based A* algorithm was applied in the first level for finding the geometric path and select multiple sub-goals path points for nest level. The paper applied the Least Squares Strategy Iteration (LSPI) into the second level, which was an approximate strategy iteration algorithm, to generate a smooth path under the restriction of robots' kinematics. ${ }^{[11]}$. Mihai et al. applied neural networks together with reinforcement leaning into a new approach to solve the problem of robots in environments with static and dynamic obstacles. This approach helped people to take control of the 
robots at any speed, and avoid local minima and converges in complex environments $^{[12]}$.

In recent years, researchers have also made great progress in modifying those methods above. Luviano et al. (2017) modified the classic algorithms of multi-agent so that it does not need the unvisited state. With estimating unknown environment, the paper applied the neural kernel smoothing and networks to the approximate greedy actions, and verified the feasibility of this modified algorithm with experiments and simulation $^{[13]}$. Zhuang et al. (2020) proposed a digital twin-based assembly data management and process traceability approach for complex products, namely Digital Twin-based Assembly Process Management and Control System (DT-APMCS), which can verify the proposed approach efficiency ${ }^{[14]}$. Saeed et al. (2020) generated the collision-free path with grid models and potential functions. In order to find the initial feasible path and avoiding obstacles, they developed a boundary node method and a path enhancement method. The above two methods were proved to be feasible by testing in work environments in different degree of complexity ${ }^{[15]}$.

\section{B. Reinforcement learning}

As one of the machine learning methods, reinforcement learning is used to describe and solve the learning strategies problem to achieve goals or maximize returns in the process of interaction between the agent and the surrounding environment. Samuel introduced the concept of reinforcement learning into the game of checkers, and used a value function represented by a linear function to guide the selection ${ }^{[16]}$. After years of research, the development of reinforcement learning becomes rapid. More and more algorithms are proposed, among which the Q-learning method has been widely used.

Recently, more and more modifications are applied to Q-learning. Konar, A et al. (2013) provided a new deterministic Q-learning with a presumed knowledge, which 
used four derived properties to update the entries in the Q-table in one time. Compared with the traditional Q-learning approach, this modified method had less time complexity and smaller required storage ${ }^{[17]}$. Robots sometimes face the problems of slow convergence speed and long-planned path when they are in an unknown environment with applying the Q-Learning method to perform path planning. Aiming to solve these problems, Meng Zhao et al.(2020) propose the Experience-Memory Q-Learning (EMQL) algorithm, which continuously updates the shortest distance from the current state node to the start point ${ }^{[18]}$. Chunyang $\mathrm{Hu}$ et al.(2020) also introduced an experience aggregative reinforcement learning method with a Multi-Attribute Decision-Making (MADM) to complete the mission of avoiding the real-time obstacle by decomposing the original obstacle avoidance task into two separate sub-tasks. Each sub-task is trained individually by a double Q-learning with a simple reward function. This method can solve the problem that the robots can hardly choose an appropriate action in the control task result from the exploration-exploitation dilemma ${ }^{[19]}$.

\section{Digital Twin Framework for assembly}

Digital twin system integrates information control system and hardware implementation system including man-machine. It provides a new virtual assembly mode for industrial production, including virtual control environment and actual physical system. The integration of computing process and physical process, in order to maintain the space-time consistency of virtual model and equipment entity in the process of motion, provides a strong support for improving the robot path planning in unknown environment and reducing the error between virtual and actual assembly. So as to improve the application efficiency of virtual assembly in the actual industrial production. And it is more convenient for six axis industrial robot and other new 
robots to be widely used in industrial production.

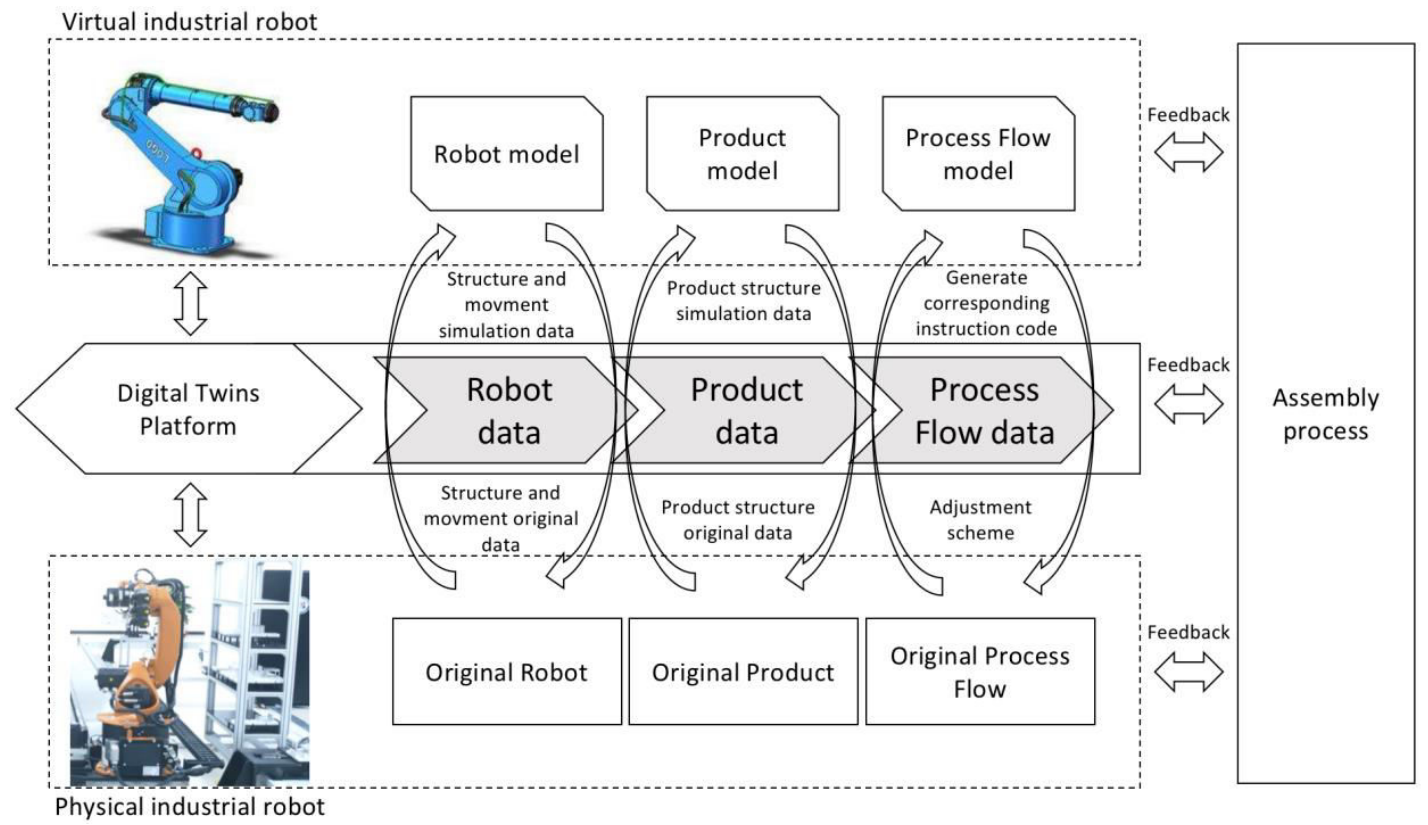

Figure 1 Digital Twin Framework for assembly

The sensing layer contains several measuring, feedback and assembly devices, which have sensing, communication and executing abilities respectively. The sensing device, such as grating ruler, laser sensor and vision sensor, sends the timely measurement data to the monitor after sensing the status attribute information concerned by the user; the execution device, including the flexible assembly part on the assembly robot as well as the whole digital production line, completes the corresponding operations according to the received instructions, such as adjusting the posture, drilling, trimming and so on. All these data can be transmitted through the network. The ability of sensing and controlling the physical elements is the basis of realizing the interaction with the information system.

The model library together with the perception of the underlying data constitute the assembly situation, including the data of each joint of the assembly robot, and the position and size data of each product. Through matching and knowledge reasoning the assembly task and situation, the corresponding assembly process schemes are 
formed. Based on that the needed assembly resources are obtained. The algorithm library sequentially generates the corresponding instruction code and adjustment scheme to drive the assembly equipment. During the real-time operation of the assembly equipment, the feedback equipment receives the corresponding feedback data and interacts with the simulation results. This off-line pre-assembly process and CPS on-line simulation control further ensures the smooth assembly.

IV. Theoretical Framework

a) Q-learning

Q-learning is a value-based algorithm in reinforcement learning. $Q$, also represented as $Q(s, a)$, is the obtainable feedback when taking action $a$, under a certain state $s$. The main objective of this algorithm is to get the optimal Q value through iteration. A Q-table will be created to reserve the $Q$ value. The agent will select an action a based on the $\varepsilon$-greedy theory under a randomly given state s. After taking the given action, a new state s' will be generated, and a reward $r$ will be given from the outside environment. The reward value will then be reserved in a matrix $(\boldsymbol{R})$ for later calculation of $\mathrm{Q}$ value in next step. After using the reward to calculate the $\mathrm{Q}$ value, the $\mathrm{Q}$ value will then be reserved in a new matrix, called $\boldsymbol{P}$. Finally, the action sequence will be generated based on the optimal (i.e. maximum) $Q$ value. The detailed algorithm is shown below.

Table 1

Initialize $Q(s, a)$ arbitrarily

Repeat (for each episode):

Initialize $s$

Repeat (for each step pf episode): 
Choose $a$ from $s$ using policy derived from $Q$ (i.e.

$\varepsilon$-greedy)

Take action $a$, observe $r, s^{\prime}$

$Q(s, a) \leftarrow Q(s, a)+\alpha\left[r+\gamma \max _{a^{\prime}} Q\left(s^{\prime}, a^{\prime}\right)-Q(s, a)\right]$

$s \leftarrow s^{\prime}$

until $s$ is terminal.

In this algorithm, s is the current state, while s' is the next state. a and a' represent the current action and next action. $Q(s, a)$ is the corresponding value of $\mathrm{s}$ and $\mathrm{a} . \mathrm{r}$ is the corresponding reward. $\gamma(0<\gamma<1)$ is a factor that reflect the future feedback on current situation. $\alpha$ represents the learning efficiency.

b) Dual reward in improved Q-Learning

In Q-Learning, the reward function plays an important part. It could help the agent to better find the right way towards the goal. With a well-designed reward function, agent can find the optimal way in a short time with least steps. In most of the cases, the goal node is represented by an especially large reward value, while the common nodes that contains no goal and obstacles will only have a small positive number or even zero. The nodes that represent obstacles will have a negative number, which can prevent the conflict between agent and obstacles. However, with this kind of static reward function, the agent will be easily trapped in blind searching. Therefore, in this paper, a dual reward function, which include static reward function and dynamic function, will be introduced.

- The static reward function

In most of the cases, the state nodes can be represented by obstacles nodes, common nodes and goal node. In this research, the reward function only contains two different 
kinds of nodes, common nodes and goal nodes. And the reward function is shown below.

$$
\operatorname{Reward}_{s}=\left\{\begin{array}{c}
0, \text { if the node is the common node } \\
10, \text { if the node is the target node }
\end{array}\right.
$$

- The dynamic reward function

In an unknown environment, the robot with static reward function can be easily trapped in blind searching. For example, when the potential next state nodes of the robot are all common nodes, which means the robot will not be punished or highly rewarded no matter what action it takes in the next state. It can choose the action of any direction, or even backwards, which will increase the distance between the robot and the goal node. Therefore, the dynamic reward function is applied to calculate the distance between its current node and the target node. The dynamic reward function is shown below.

$$
\begin{gathered}
d_{t}=\sqrt{\left(x_{\text {target }}-x_{t}\right)^{2}+\left(y_{\text {target }}-y_{t}\right)^{2}} \\
d_{t+1}=\sqrt{\left(x_{\text {target }}-x_{t+1}\right)^{2}+\left(y_{\text {target }}-y_{t+1}\right)^{2}} \\
\text { Reward }_{d}=\lambda \frac{d_{t}-d_{t+1}}{\left|d_{t}-d_{t+1}\right|}
\end{gathered}
$$

In these equation, $d_{t}$ represents the distance between current state node and target node. And the $d_{t+1}$ represents the distance between next state node and target node. $\lambda$ is a factor that depends on static reward, since it represents the proportion of dynamic reward in the total reward the agent would get. In this research, we give the value of 0.1 to $\lambda$.

c) Q-table optimized by case-based reasoning

In the training process of Q-learning, the initial parameter values are difficult to 
choose. The reward matrix in the $\mathrm{Q}$ learning algorithm is a constant matrix, and the initial Q-table is often initialized to a zero-valued matrix. Therefore, it is more sensitive to the parameter values in the algorithm in the early stage of the algorithm and is often directly given based on empirical values. However, the same initial parameter values cannot be applied to networks of different scales and densities, so multiple experiments are required in different networks to determine the parameter values. Moreover, large space is necessary for exploration. At the beginning of the algorithm, under the guidance of lack of prior knowledge, the agent has been in the random exploration stage for a long time, and it is difficult to quickly approach the optimal solution. At the same time, random exploration may cause the algorithm to converge to the local optimal value in advance, and under the positive feedback effect of the roulette exploration strategy, the degree of exploration of the sub-optimal path is strengthened, which causes the algorithm to fail to jump out of the local optimal. In order to make better use of the experience of historical cases, we use case-based reasoning methods to initialize q-table to improve the speed and quality of algorithm training.

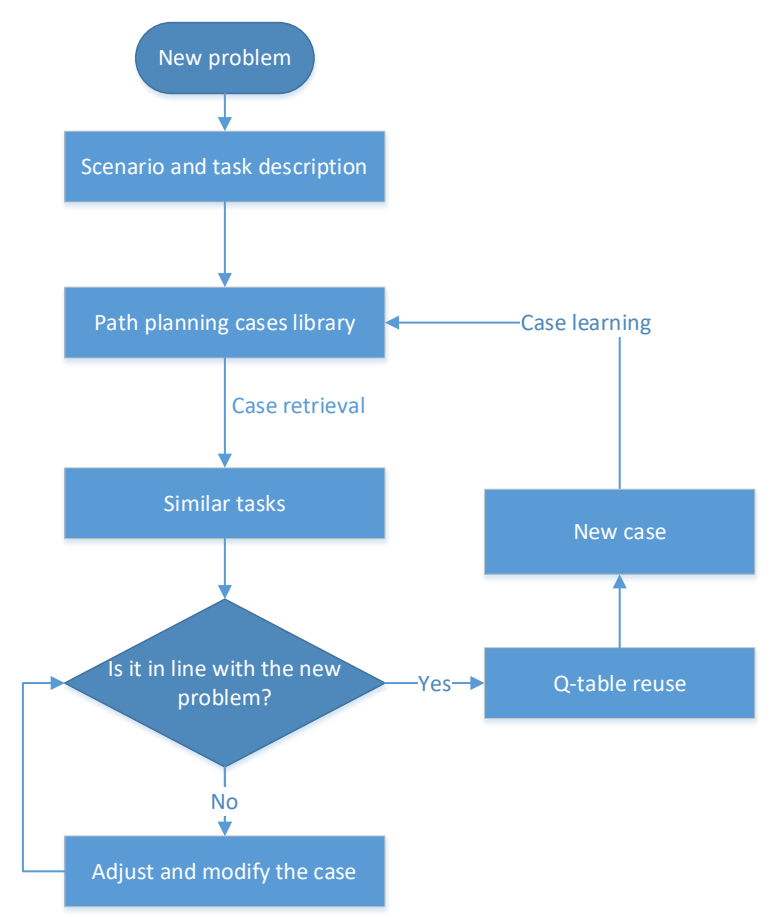


Figure 2 Flow chart of Q-table optimization based on CBR

The case-based reasoning method has the function of analogical learning, which relies on past experience for learning and problem solving. A case library is constructed to store historical path planning cases. The solution of the new case can be obtained by modifying the case in the case library that is similar to the current situation. First, obtain the information used or generated in path planning. Each case in the case library contains information such as the task, environment, and path generated during each path planning. Each case can be obtained through a specific index, this index value is generated by the current planning task, environment and other information. By matching the context and task of the current task with the cases in the library, and evaluating and correcting the matched candidate cases, the $\mathrm{Q}$ table used by the case with the best performance is finally used as the initial Q table of the current task. At the same time, the case-based planning method continuously expands and updates the case library through iterative learning. In this study, the calculation of task similarity is as follows:

$$
S\left(\pi_{1}, \pi_{2}\right)=\frac{p\left(\pi_{1} \cap \pi_{2}\right)}{\max \left(p\left(\pi_{1}\right), p\left(\pi_{2}\right)\right)}
$$

where, $\pi_{1}, \pi_{2}$ respectively represents the collection of obstacle points in the two scenes. $p\left(\pi_{1} \cap \pi_{2}\right)$ represents the number of point sets in the same area covered by obstacles in two scenes, and $\max \left(p\left(\pi_{1}\right), p\left(\pi_{2}\right)\right)$ represents the number of point sets in obstacles with a larger coverage area.

d) Obstacle avoiding method

In some of the cases, a large negative reward would be set for the obstacle nodes in the reward function in order to keep robot from encountering the obstacles. However, this research try to record down the obstacle nodes, and find the feasible actions and states at first every time the agent is going to move on to the next state. With this 
method, the possibility of hitting a obstacle can be reduced comparing to the method of giving the obstacle nodes a large negative rewards, since the Q-Learning method will sometimes choose actions and states randomly, ignoring the value of reward.

\section{Experience and analysis}

In order to verify the theoretical analysis effect of path planning based on the improved Q-learning algorithm proposed in the previous section, the manipulator and its working environment in the assembly digital twin system were modeled and verified. For specific assembly tasks, the end of the six-joint industrial robot needs to start from the starting position coordinate point in its working space, avoid possible obstacles in the working space, and plan the path to finally reach the target coordinate point. This study selects the path planning scenario shown in the figure below, and takes a typical six-joint robot UR10 as an example to explore its performance in pathfinding in the below scenario. The virtual environment contains obstacles, targets in the shape of rectangular solid. Although most of the obstacles and targets in reality are in different irregular shape, we modified them into rectangular solid for convenient calculation. The constructed virtual environment is shown below. The software and hardware environments for system implementation are summarized as follows: (1) Application server: A ThinkPad desktop with Intel Core i7 CPU (3.40 GHz) and 32GB memory, 2T SCSI HD and a Windows Server operating system; (2) Programming platform: PyCharm and Python 3.6.0; (3) Database: MySQL 6.0; (4) Network configuration: $1 \mathrm{G}$ Ethernet network connection between testing clients and application server. 

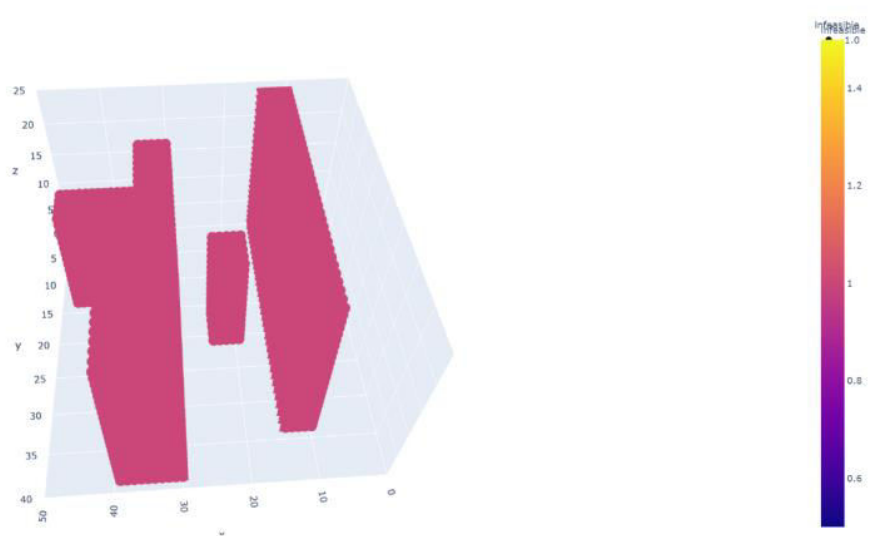

Figure 3 Path planning environment with obstacles

The actual maximum working range of the UR10 manipulator is a $1300 \mathrm{~mm}$ radial range. Considering the flexibility and safety of the actual assembly task of the robot, the working range is selected to be a three-dimensional space of $250 \mathrm{~mm} \times$ $200 \mathrm{~mm} \times 125 \mathrm{~mm}$. Taking $5 \mathrm{~mm}$ as the minimum unit for the end of the robot to move, the three-dimensional workspace of the robot can be divided into a discrete state set containing $50 \times 40 \times 25$ grids $\boldsymbol{S}$, starting point $\mathrm{H}(0,0,0)$, ending point $\mathrm{T}$ $(32,30,12)$. The pink area is the obstacles such as the workbench in the environment, and the white area is the feasible region for the robot to work freely.

The robot's possible motion directions include up, down, front, back, left, right, and a combination of the three coordinate directions. Therefore, the action set A of the robot includes basic element actions in 26 directions. By setting equal algorithm parameters, set $\lambda$ from 0 every 0.5 to 1 to perform learning algorithms that without the improved dynamic reward function and prior scene information, as well as the learning algorithm that introduces the dynamic reward function, and the original Q learning algorithm. Simulation experiment comparison. Set the maximum number of iterations to 10000 , and observe the number of steps and the number of steps and the number of iterations experienced when the planned path stabilizes during the process of planning the path from the start to the end of the robot. 


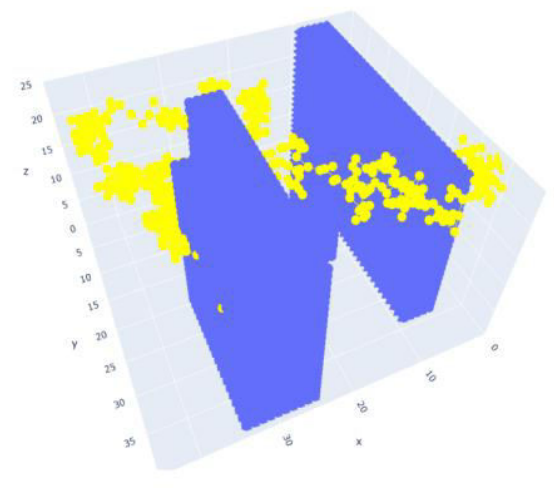

a) original Q-learning, iteration $=200$

b) original Q-learning, iteration $=2000$

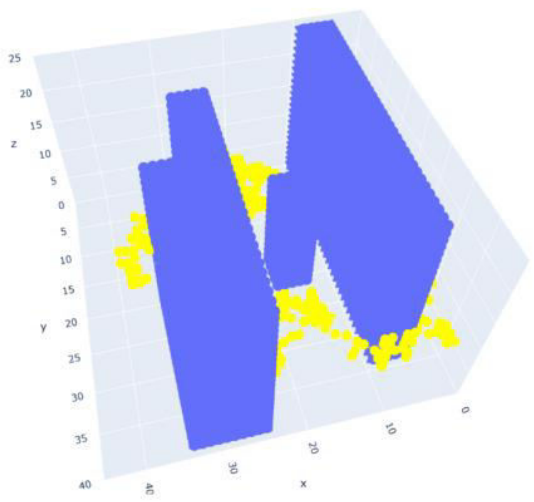

c) dynamic reward, iteration $=200$

d) dynamic reward, iteration $=2000$
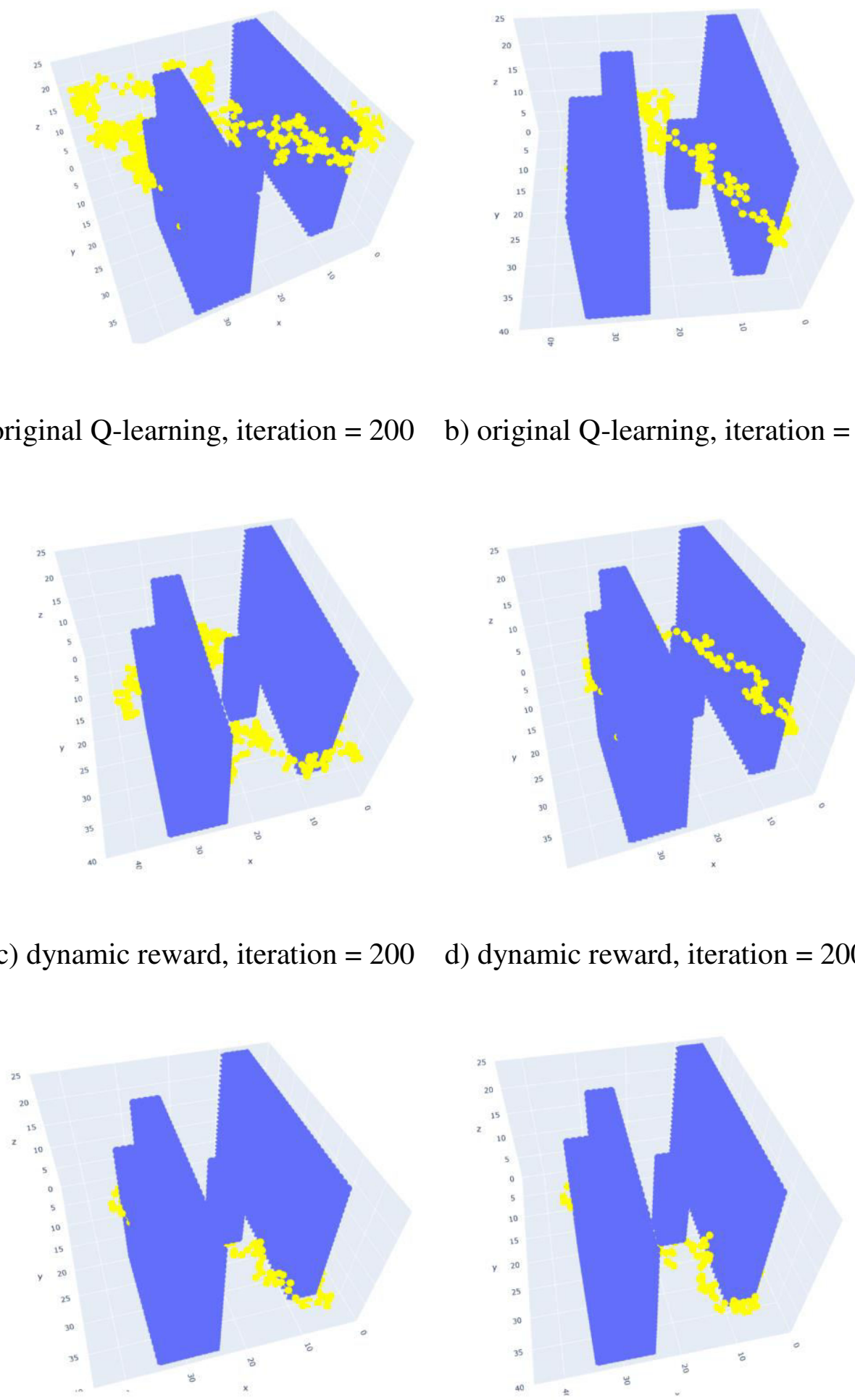

f) dynamic reward and CBR, iteration $=2000$

Figure 3 Planning paths under different algorithms and iterations 
Figure 3 shows the path of the manipulator in the initial and final stages of different algorithms. In the scene where the robot has obstacle areas, especially trap areas, using appropriate profit gain and environmental prior information can improve the efficiency of algorithm optimization, and iterative steps are fewer. Although the round update makes the update time of each step longer, after fewer rounds of update, the iterative step reduction is more advantageous, making the learning path of the improved algorithm shorter and the overall efficiency of the algorithm better. For the modified Q-Learning method in this research, dual reward function, CBR and obtaining feasible action method are applied. Both of these methods can help the robot find optimal route towards the target in a safer and more efficient way. For the dual reward function method, instead of the original reward function, this research combines both the static reward and dynamic reward as a whole. The former reward can help the robot identify the status of next action, while the latter reward can lead the robot to the right direction towards the target. For the obtaining feasible action method, the robot can avoid the collision with obstacles, and the safety level of this method is much higher than simply setting the obstacle nodes with large negative value as static reward.

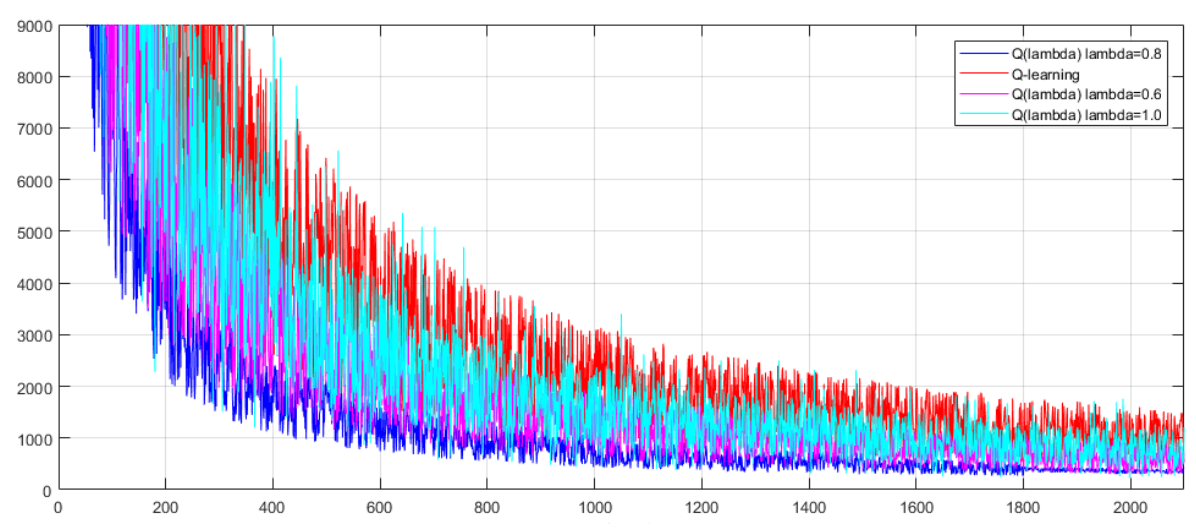

Figure 4 Number of iterations per round under different $\lambda$ 
Furthermore, when the improved Q learning algorithm takes three values of 0.6, 0.8, and 1 in $\lambda$, the algorithm compares and observes the steps required for each iteration of the pathfinding learning process. The index of the iteration round is taken as the horizontal axis, and the pathfinding steps of the robot in each round are taken as the vertical axis. The comparison of step changes is shown in Figure 4. In fact, when $\lambda$ is set to 0 , the learning algorithm becomes the original Q learning algorithm. Therefore, if $\lambda$ is too small, the effective income of the update will be annihilated more quickly. If $\lambda$ is too large, it will cause a certain number of repeated steps to explode. Locally optimal, so the iterative effect of some rounds does not converge, and the movement is repeated in the trap area.

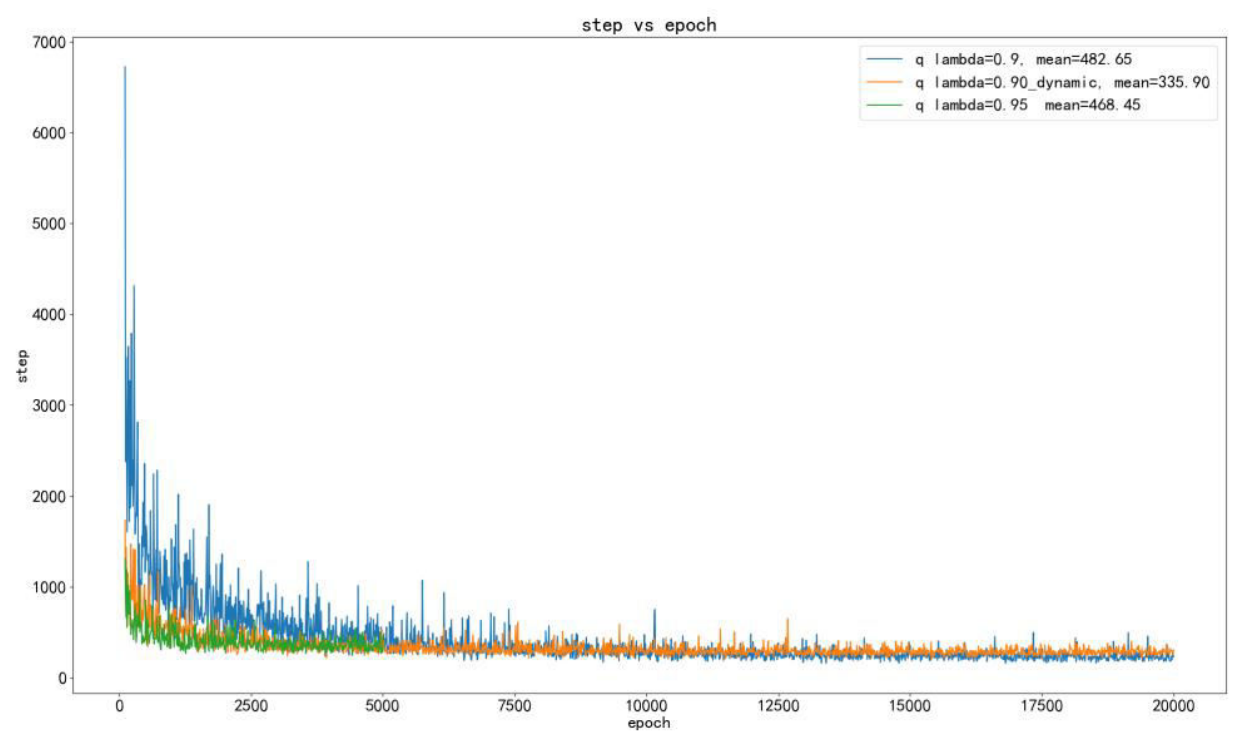

Figure 5 Steps per epochs of different algorithms

Figure 5 shows the steps per epochs of different algorithms. Q learning algorithm, $\mathrm{Q}$ algorithm with dynamic reward function, and $\mathrm{Q}$ algorithm with dynamic reward function and CBR converges at 6000 epochs, 2500 epochs, and 2000 epochs respectively, when the planned path and number of iterations stabilized, and the algorithm steps decreased significantly faster. The Q learning algorithm still does not converge when iterates 2000 epochs, and the algorithm's pathfinding performance at 
2000 iterations is worse than the former at about 1500 iterations, and the pathfinding performance at 1000 iterations is the same as that of the former. The effect of 200 iterations is equivalent, so in the scene with trap area obstacles, the pathfinding performance of the $\mathrm{Q}$ learning algorithm with dynamic reward function and CBR is significantly better than the Q learning algorithm.

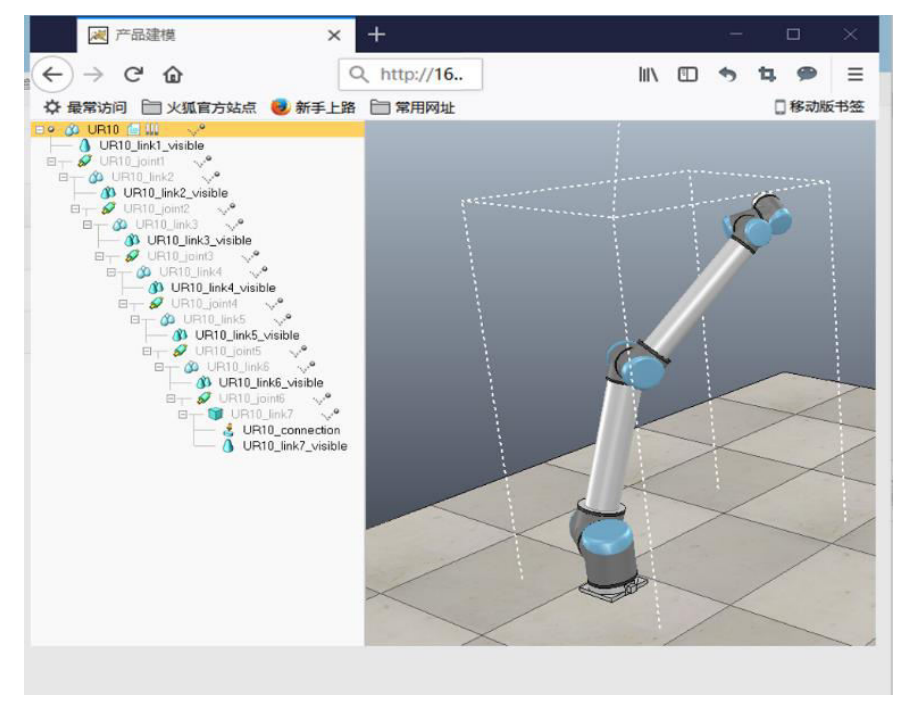

Figure 6 The GUI of manipulator modeling

In order to realize the centralized management of distributed resources by the cloud service architecture and the assembly requirements of centralized resources for decentralized services, a prototype system was developed using the web-based B/S (Browser/Server) architecture model. Figure 6 shows an example of editing UR10 in the equipment model library shown in the integrated Vrep modeling software.

\section{Conclusions}

The assembly path planning of manipulators is playing an increasingly important role in modern manufacturing. The digital twin system can provide an intelligent assembly paradigm, which realizes the real-time synchronization of virtual assembly and actual assembly through the integration of intelligent calculation process and physical process. In this research, a digital twin system for manipulator assembly is constructed, which includes functions such as real-time perception, data exchange, communication, intelligent decision-making, and virtual-real interaction. Moreover, 
this research also proposes a modified Q learning algorithm, which speeds up the convergence speed by adding dynamic reward function, optimizes the initial Q table by introducing knowledge and experience through the CBR algorithm, and prevents entry into the trapped area through the obstacle avoiding method. Finally, take the six-joint robot UR10 as an example to verify the performance of the algorithm in the three-dimensional space of pathfinding. The experimental results show that the improved Q-learning algorithm's pathfinding performance is significantly better than the Q-learning algorithm.

As far as future research is concerned, there is great potential for the extension of the current model and solution algorithm. The proposed algorithm can be improved from the following aspects: in theoretical research, a more in-depth discussion on specific scenario modeling methods and control algorithms is still needed; application exploration also needs to be expanded from more perspectives, and the research on system architecture needs to be more detailed.

\section{Declarations}

a. Funding: This research is supported by the Fundamental Research Funds for the Central Universities under the grant number FRF-TP-20-050A2.

b. Conflicts of interest/Competing interests: No conflict of interest exists, or if such conflict exists, the exact nature of the conflict must be declared and if accepted.

c. Availability of data and material (data transparency): The datasets used or analyzed during the current study are available from the corresponding author on reasonable request.

d. Code availability (software application or custom code): The code used during the current study are available from the corresponding author on reasonable request.

e. Ethics approval (include appropriate approvals or waivers): The article has no ethics problem 
f. Consent to participate (include appropriate statements): the article is original, has been written by the stated authors who are all aware of its content and approve its submission

g. Consent for publication (include appropriate statements): this article has not been published previously. The article will not be published elsewhere in the same form, in any language, without the written consent of the publisher.

\section{References}

[1] Kumar, K., Zindani, D., \& Davim, J.P. (Eds.). (2019). Digital Manufacturing and Assembly Systems in Industry $4.0 \quad$ (1st ed.). CRC Press. https://doi-org.libproxy1.usc.edu/10.1201/9780429464768

[2] Guo, F., Cai, H., Ceccarelli, M., Li, T., \& Yao, B. (2020). Enhanced D-H: An improved convention for establishing a robot link coordinate system fixed on the joint. The Industrial Robot, 47(2), 197-205. doi:http://dx.doi.org.libproxy1.usc.edu/10.1108/IR-09-2019-0185

[3] Polini, W., \& Corrado, A. (2020). Digital twin of composite assembly manufacturing process. International Journal of Production Research, 58(17), 5238-5252. https://doi.org/10.1080/00207543.2020.1714091

[4] Lu, Yang. "Cyber physical system (CPS)-based industry 4.0: A survey." Journal of Industrial Integration and Management 2.03 (2017): 1750014.

[5] Speicher, C., Vette, M., Hörauf, L., \& Müller, R. (2016). Identification of Assembly System Configuration for Cyber-Physical Assembly System Planning. Applied Mechanics and Materials, 840(Robotics and Automated Production Lines), 24-32. https://doi.org/10.4028/www.scientific.net/AMM.840.24

[6] Sutton, R. S., \& Barto, A. G. (2018). Reinforcement learning: an introduction (Second edition.). The MIT Press. 
[7] Beltran-Hernandez, C. C., Petit, D., Ramirez-Alpizar, I. G., \& Harada, K. (2020). Variable Compliance Control for Robotic Peg-in-Hole Assembly: A Deep-Reinforcement-Learning Approach. Applied Sciences, 10(19), NA. https://link.gale.com/apps/doc/A640017582/GRNR?u=usocal_main\&sid=bookm ark-GRNR\&xid=edb15a0e

[8] Khatib, Oussama.Real-Time Obstacle Avoidance for Manipulators and Mobile Robots[J].International Journal of Robotics Research,1986,Vol.5(1): 90-98

[9] Tsong-Li Lee (1) ;Chia-Ju Wu (2) .Fuzzy Motion Planning of Mobile Robots in Unknown Environments[J].Journal of Intelligent and Robotic Systems,2003,Vol.37(2): 177-191

[10]Zuo, L., Guo, Q., Xu, X., \& Fu, H. (2015). A hierarchical path planning approach based on $\mathrm{A} *$ and least-squares policy iteration for mobile robots. Neurocomputing, 170, 257-266.

[11]Duguleana, Mihai 1 , (mihai.duguleana@unitbv.ro);Mogan, Gheorghe 2 , (mogan@unitbv.ro).Neural networks based reinforcement learning for mobile robots obstacle avoidance[J].Expert Systems with Applications,2016,Vol.62: $104-115$

[12]Luviano Cruz, D. 1 ;Wen Yu 1 .Path planning of multi-agent systems in unknown environment with neural kernel smoothing and reinforcement learning[J].Neurocomputing,2017,Vol.233: 34-42

[13]Zhuang C , Gong J , Liu J . Digital twin-based assembly data management and process traceability for complex products[J]. Journal of Manufacturing Systems, 2020.

[14]Saeed, R.A. 1 (AUTHOR)( raza.saeed@ unica.it);Recupero, Diego Reforgiato 1 (AUTHOR)(_diego.reforgiato@unica.it);Remagnino, Paolo 2 (AUTHOR)( p.remagnino@kingston.ac.uk).A Boundary Node Method for path planning of mobile robots.[J].Robotics \& Autonomous Systems,2020,Vol.123 
[15] Samuel, A. L..Some studies in machine learning using the game of checkers.[J].IBM Journal of Research and Development,1959,Vol.3: 211-229

[16]Konar, A(Konar, Amit) [1] ;Chakraborty, IG(Chakraborty, IndraniGoswami) [1] ;Singh, SJ(Singh, SapamJitu) [1] ;Jain, LC(Jain, LakhmiC.) [2] ;Nagar, AK(Nagar, AtulyaK.) [3] .A Deterministic Improved Q-Learning for Path Planning of a Mobile Robot[J].Systems, Man and Cybernetics: Systems, IEEE Transactions on,2013,Vol.43(5): 1141-1153

[17]Meng Zhao;Hui Lu;Siyi Yang;Fengjuan Guo.The Experience-Memory Q-Learning Algorithm for Robot Path Planning in Unknown Environment[J].IEEE Access,2020,Vol.8: 47824-47844

[18]Chunyang Hu;Bin Ning;Meng Xu;Qiong Gu.An Experience Aggregative Reinforcement Learning With Multi-Attribute Decision-Making for Obstacle Avoidance of Wheeled Mobile Robot[J].IEEE Access,2020,Vol.8: $108179-108190$ 